\title{
Spectral modeling of rotating turbulent flows
}

\author{
J. Baerenzung, ${ }^{1}$ P. D. Mininni, ${ }^{1,2}$ A. Pouquet, ${ }^{1}$ H. Politano, ${ }^{3}$ and Y. Ponty ${ }^{3}$ \\ ${ }^{1}$ TNT/NCAR, P.O. Box 3000, Boulder, Colorado 80307-3000, USA \\ ${ }^{2}$ Departamento de Física, Facultad de Ciencias Exactas y Naturales, Universidad de Buenos Aires, \\ Ciudad Universitaria, 1428 Buenos Aires, Argentina \\ ${ }^{3}$ Laboratoire Cassiopée, UMR 6202, Observatoire de la Côte d'Azur, B.P. 4229, 06304 Nice Cedex 4, \\ France and CNRS UMR 6202, Observatoire de la Côte d'Azur, Université de Nice-Sophia Antipolis, \\ B.P. 4229, 06304 Nice Cedex 4, France
}

(Received 10 December 2008; accepted 7 December 2009; published online 24 February 2010)

\begin{abstract}
A subgrid-scale spectral model of rotating turbulent flows is tested against direct numerical simulations (DNSs). The case of Taylor-Green forcing is considered, a configuration that mimics the flow between two counter-rotating disks as often used in the laboratory. Computations are performed for moderate rotation down to Rossby numbers of 0.03 , as can be encountered in the Earth's atmosphere. We provide several measures of the degree of anisotropy of the small scales and conclude that an isotropic model may suffice at moderate Rossby number. The model, developed previously [J. Baerenzung, H. Politano, Y. Ponty, and A. Pouquet, "Spectral modeling of turbulent flows and the role of helicity," Phys. Rev. E 77, 046303 (2008)], incorporates eddy viscosity and eddy noise that depend dynamically on the index of the energy spectrum. We show that the model reproduces satisfactorily all large-scale properties of the DNS up to Reynolds numbers of $\sim 10^{4}$ and for long times after the onset of the inverse cascade of energy; it is also shown to behave better than either the Chollet-Lesieur eddy viscosity model [J. P. Chollet and M. Lesieur, "Parametrization of small scales of three-dimensional isotropic turbulence utilizing spectral closures," J. Atmos. Sci. 38, 2747 (1981)] or an under-resolved DNS. () 2010 American Institute of Physics.

[doi:10.1063/1.3292008]
\end{abstract}

\section{INTRODUCTION}

Rotating flows are commonplace in nature, the influence of rotation being measured by the Rossby number Ro $=U_{0} / 2 L_{0} \Omega$, with $U_{0}$ being the rms velocity, $L_{0}=2 \pi / k_{0}$ being a characteristic length scale of the flow (associated, for example, with the forcing), and $\Omega$ being the rotation rate. The Rossby number of the atmosphere is $\sim 0.1$, and in the ocean it can be as small as $10^{-3}$. Assuming a constant rotation rate, the Coriolis force that appears in the equations leads to the emergence of wave motions, which, at small enough Rossby number, can be thought as dominating the dynamics. However, at high Reynolds number, $\operatorname{Re}=U_{0} L_{0} / \nu$, with $\nu$ being the viscosity, turbulent eddies interact with waves, and inertial waves interact nonlinearly (in particular through resonances) so that the dynamics becomes complex. The Rossby number can be viewed in this way: as the ratio of the characteristic time of an inertial wave, $\tau_{W} \sim 1 / \Omega$, to the characteristic time of an eddy, $\tau_{\mathrm{NL}} \sim L_{0} / U_{0}$; when Ro is small, the waves are rapid and may dominate the dynamics.

Many studies have been devoted to the exploration of rotating turbulence, experimental as well as numerical and theoretical (see, e.g., Ref. 1 and references therein). One expects the flow to become quasibidimensional under the influence of strong rotation, but recent studies show that the dynamics is more subtle, with three-dimensional eddies possibly prevailing at small scales. The case of small Rossby number can be studied using either weak turbulence ${ }^{2}$ or anisotropic extensions of closure models, such as the eddy damped quasinormal Markovian (EDQNM hereafter) ap- proximation. In such approaches, the closure is obtained either by using resonances or by modeling the damping of fourth-order cumulants (nonzero for a non-Gaussian field) by a term linear in third-order moments; dimensionally, the constant of proportionality is the inverse of a time, or a rate $\mu$, taken in EDQNM to be the rates known to be significant in the physics of the problem. In the simplest case of nonrotating isotropic and homogeneous turbulence, these rates are proportional to the inverse of the local eddy turn-over time $\tau_{\ell}=\ell / U_{\ell}$ and of the viscous time $\tau_{D} \sim \ell^{2} / \nu$, expressed in terms of the scale $\ell$ and the velocity at that scale $U_{\ell}$. In the rotating case, the wave frequency becomes relevant as well ${ }^{3}$ (see Ref. 4 for an early realization of this concept), and because of the anisotropic dispersion relation of inertial waves, the model becomes anisotropic itself in terms of a spectral energy distribution that is a function of the wavenumbers $k_{\perp}$ and $k_{\|}$, where $\perp$ and $\|$ refer to directions relative to the rotation axis.

In a forced flow, high resolutions and long-time integrations are needed in order to resolve the different spatial regimes that may develop (e.g. inverse and direct cascades of energy $^{5-9}$ and direct cascades of helicity ${ }^{10}$ ), as well as the short-time wave regime versus the long-time turbulent regime. Modeling becomes a necessity in order to reach an understanding of these flows at high Reynolds numbers as what occurs in astrophysics and geophysics. Using a closure set of integrodifferential equations for the energy spectra (see Ref. 11 and references therein) or the weak turbulence framework developed in Ref. 2 is a powerful tool for sufficiently small Rossby number, but two difficulties have to be 
overcome. On the one hand, such techniques are valid in the limit $\mathrm{Ro} \rightarrow 0$, and yet the flows one tries to model may present inhomogeneities (in space, in time, and in scale) that are affected differently by the rotation. On the other hand, the complexity of the so-called weak turbulence kinetic equations and in particular their dependence on the angle between the wavevector (in a Fourier decomposition) and the rotation axis (to be taken as the $z$ axis in what follows) necessitates a regular discretization in angle as opposed to an exponential discretization in wavenumber, the latter working because of the self-similarity of the known (power law) spectral solutions to the equations. This angular dependency makes the closure of weak turbulence equations difficult and costly to use (see however Ref. 11). Another model, based on the dynamics of the second-order structure function of the velocity field and without adjustment of the model coefficients, ${ }^{12}$ can be built for anisotropic flows; it shows reasonable agreement with data when compared to rotating decaying flows in the absence of viscosity.

As previously mentioned, it has been shown by several authors that the expected bidimensionalization of the flow is only realized partially, and small-scale eddies may not follow such dynamics; in this case, one expects the small-scale eddies [i.e., those that are to be modeled in an large eddy simulation (LES) approach] to be isotropic to some extent. It may thus be envisageable to use, as a model of small unresolved scales, a methodology developed for isotropic flows. It is in this context that we extend the study of the spectral model derived in Ref. 13 to the case of forced rotating flows, comparing the results of the model to direct numerical simulations (DNSs) (Ref. 9) for forced rotating turbulence down to Ro $\sim 0.03$. The model is based on the EDQNM closure to compute eddy viscosity and eddy noise. It adapts dynamically to the inertial index of the energy spectrum, and as a result it is well suited to study rotating turbulence for which the scaling laws are not well known, and may change with the Rossby number, or even (at fixed Rossby number) as the system evolves and an inverse cascade develops. The next section poses the problem in terms of equations and models and gives the numerical setup. We then describe the results for the isotropic LES model, examining energetic balance, measures of anisotropy, structures, spectra, and higherorder statistics; we also present in Sec. IV D an intercomparison with the Chollet-Lesieur (CL) model ${ }^{14}$ as well as with an under-resolved DNS. Finally, Sec. V presents our conclusions.

\section{EQUATIONS AND SPECTRAL MODELING}

\section{A. Primitive equations}

The dynamical equations can be written in terms of the Fourier coefficients of the velocity field defined as usual as

$$
\mathbf{v}(\mathbf{k}, t)=\iiint_{-\infty}^{\infty} \mathbf{v}(\mathbf{x}, t) e^{-i \mathbf{k} \cdot \mathbf{x}} \mathbf{d} \mathbf{x} .
$$

In the rotating frame and including the centrifugal force in the pressure term, the equations are

$$
\left(\frac{\partial}{\partial t}+\nu k^{2}\right) v_{\alpha}(\mathbf{k}, t)+2 \Omega P_{\alpha \beta} \varepsilon_{\beta z \gamma} u_{\gamma}(\mathbf{k}, t)=t_{\alpha}(\mathbf{k}, t)+F_{\alpha}(\mathbf{k})
$$

together with incompressibility $(\mathbf{k} \cdot \mathbf{v}=0) ; \nu$ is the kinematic viscosity, $\mathbf{F}(\mathbf{k})$ is the Fourier transform of the forcing function, $P_{\alpha \beta}=\delta_{\alpha \beta}-k_{\alpha} k_{\beta} / k^{2}$ is the projection operator, $\Omega$ is the rotation rate, and $\mathbf{t}(\mathbf{k}, t)$ is a bilinear operator for the kinetic energy transfer written as

$$
t_{\alpha}(\mathbf{k}, t)=-i P_{\alpha \beta}(\mathbf{k}) k_{\gamma} \sum_{\mathbf{p}+\mathbf{q}=\mathbf{k}} v_{\beta}(\mathbf{p}, t) v_{\gamma}(\mathbf{q}, t) .
$$

Note that $P_{\alpha \beta}$ is a projector that allows us to take the pressure term of the velocity equation into account via a Poisson formulation and ensures that the velocity remains divergence-free including in the presence of rotation. Finally note that the total energy $E_{T}=\left\langle\mathbf{v}^{2} / 2\right\rangle$ and the helicity $\langle\mathbf{v} \cdot \omega\rangle$ (with $\omega=\nabla \times \mathbf{v}$ ) are invariants of the three-dimensional equations in the ideal case, i.e., in the absence of viscous dissipation $(\nu=0)$. Besides the Reynolds number and the Rossby number defined previously, one can also introduce dimensionless numbers based on small scales as produced by the turbulent flow; the simplest way to do that traditionally is to base such parameters on the vorticity through the Taylor scale $\lambda$ defined as

$$
\lambda=2 \pi\left(\frac{\int E(k) d k}{\int E(k) k^{2} d k}\right)^{1 / 2} ;
$$

the Taylor Reynolds number is then

$$
R_{\lambda}=\frac{U_{0} \lambda}{\nu} .
$$

One can also define a quantity called the micro-Rossby number, ${ }^{8}$ which is useful to determine the regime of the small-scale turbulence and the slope of the energy spectrum. ${ }^{15}$ It reads

$$
\operatorname{Ro}_{\omega}=\frac{\omega_{\mathrm{rms}}}{2 \Omega},
$$

where $\omega_{\text {rms }}$ stands for the rms vorticity; note that it is proportional to the Rossby number evaluated at the Taylor scale. The DNSs (runs Id, IId, and IIId, respectively; see Table I) used in this paper in order to assess the validity of the LES are those labeled A3, A4, and A6, respectively, in Ref. 9 (hereafter, Paper I). For all these runs, the forcing function is a Taylor-Green (TG hereafter) vortex with amplitude $F_{0}$ and with $k_{0}=2$,

$$
\begin{aligned}
& F_{x}=F_{0} \sin \left(k_{0} x\right) \cos \left(k_{0} y\right) \cos \left(k_{0} z\right), \\
& F_{y}=-F_{0} \cos \left(k_{0} x\right) \sin \left(k_{0} y\right) \cos \left(k_{0} z\right),
\end{aligned}
$$

with $F_{z}=0$; the third component of the forcing is equal to zero, but the velocity in the $z$-direction grows through nonlinear interactions. Moreover, the forcing injects no energy in modes with $k_{z}=0$, and as a result any amplification observed in strongly rotating cases must be only due to a cascade process. Finally, the resulting flow has a small spectral anisotropy with slightly more energy in the $z$ direction, ${ }^{9}$ an 
TABLE I. Parameters of the simulations: resolution $N^{3}$, Rossby number Ro based on the forcing scale $L_{0}$ and measured at the onset of the inverse energy cascade for run I, Taylor microscale $\lambda$ and integral scale $L$, rms velocity $U_{0}=\left\langle\mathbf{v}^{2}\right\rangle^{1 / 2}$, integral Reynolds number $\operatorname{Re}=U_{0} L / \nu$, and eddy turnover time $\tau_{\mathrm{NL}}=L_{0} / U_{0} ; t_{m}$ is the final time of the computation. Note that the $r$ label in the nomenclature of the runs stands for the reduced data obtained by filtering in spectral space to $64^{3}$ points the original $256^{3}$ DNS data and that $\lambda, L, \operatorname{Re}$, and $\tau_{\mathrm{NL}}$ are evaluated at the final time of the simulation for run I, which undergoes an inverse cascade, whereas they are averaged during the stationary phase of simulations II and III, which are at higher Rossby numbers and do not undergo any significant inverse energy transfer.

\begin{tabular}{lccccccccc}
\hline \hline & $N$ & Ro & $\lambda$ & $L$ & $U_{0}$ & Re & $\tau_{\mathrm{NL}}$ & $t_{m}$ \\
\hline Id & DNS & 256 & 0.03 & 2.06 & 5.71 & 4.53 & 12920 & 1.26 & 157 \\
Ir & Filtered DNS & 64 & $\ldots$ & 2.37 & 5.71 & 4.53 & 12927 & 1.26 & $\ldots$ \\
IL & LES & 64 & $\ldots$ & 2.07 & 5.59 & 4.60 & 12857 & 1.22 & $\ldots$ \\
IId & DNS & 256 & 0.17 & 0.65 & 1.44 & 1.01 & 729 & 1.41 & 45 \\
IIr & Filtered DNS & 64 & $\ldots$ & 0.73 & 1.45 & 1.01 & 732 & 1.44 & $\ldots$ \\
IIL & LES & 64 & $\ldots$ & 0.76 & 1.49 & 1.09 & 813 & 1.36 & $\ldots$ \\
IIId & DNS & 256 & 0.35 & 0.77 & 1.47 & 1.07 & 786 & 1.36 & 45 \\
IIIr & Filtered DNS & 64 & $\ldots$ & 0.72 & 1.41 & 0.96 & 678 & 1.46 & $\ldots$ \\
IIIL & LES & 64 & $\ldots$ & 0.75 & 1.42 & 0.98 & 695 & 1.45 & $\ldots$ \\
\hline \hline
\end{tabular}

effect that is the opposite of the tendency toward twodimensionalization that develops in rotating turbulence.

The numerical computations using the above forcing are thus either DNSs of the Navier-Stokes equations with $256^{3}$ grid points or LESs on grids of $64^{3}$ points; the axis of rotation is the $z$-axis, and the flow is initially at rest. Note that the TG flow is widely used in experimental devices to study turbulence and its effect on the generation of magnetic fields, ${ }^{16}$ even though the TG vortex has no net helicity due to its symmetries; because of this latter property, the LES model used here will not include the helicity eddy viscosity derived in Ref. 13 (Paper II hereafter). The rms velocity to determine the turn-over time is measured in the turbulent steady state as stated previously, at the onset of the inverse cascade at low Rossby number, and the value given for Ro is taken at that same time. Note that the amplitude of the forcing $F_{0}$ in each simulation is increased with $\Omega$ to have $U_{0} \approx 1$ in all the runs before the inverse cascade sets in (see Ref. 9 for more details on the DNS runs).

Finally, as the issue of the direction of the energy cascade (direct and/or inverse) is an important issue in rotating turbulence, a useful diagnostic in this context is to examine the behavior of the skewness (normalized third-order moment corresponding to energy transfer) based on the velocity derivative and evaluated on a distance $r, \partial v(r)$ (or on the vertical vorticity $\omega_{z}$; see, e.g., Ref. 6), namely,

$$
S_{k}=\frac{\left\langle\left(\partial_{x} v_{x}\right)^{3}\right\rangle}{\left\langle\left(\partial_{x} v_{x}\right)^{2}\right\rangle^{3 / 2}} \text {. }
$$

\section{B. The isotropic EDQNM closure}

The LES model derived in Ref. 13 for nonrotating Navier-Stokes flows is now extended to the rotating case in its nonhelical version (LES-P of Paper II). In other words, intrinsic variations in the helicity spectra are not taken into account in the present work in the evaluation of the transport coefficients used in our LES model. The first step of the model is to employ a spectral filtering of the equations; this operation consists in truncating all velocity components at wavevectors $\mathbf{k}$ such that $|\mathbf{k}|=k>k_{c}$, where $k_{c}$ is a so-called cutoff wave number. Since the scales associated with $k_{c}$ are presumably much larger than the actual dissipative small scales in a high Reynolds number flow, one needs to model the transfer between the large (resolved) scales and the small (subgrid unresolved) scales of the flow. In order to approximate these transfer terms, the behavior of the energy spectrum after the cutoff wave number has to be estimated. We therefore define an intermediate range lying between $k_{c}$ and $3 k_{c}$, where the energy spectrum is assumed to present a power-law behavior possibly followed by an exponential decrease,

$$
E^{V}(k, t)=E_{0}^{V} k^{-\alpha_{E}^{V}} e^{-\delta_{E}^{V} k}, \quad k_{c} \leq k<3 k_{c} .
$$

The coefficients $\alpha_{E}^{V}, \delta_{E}^{V}$, and $E_{0}^{V}$ are computed at each time step through a mean square fit of the resolved energy spectrum. In a second step, one can write the following model equations (omitting forcing):

$$
\begin{aligned}
{\left[\partial_{t}+\right.} & \left.\left(\nu\left(k \mid k_{c}, t\right)+\nu\right) k^{2}\right] v_{\alpha}(\mathbf{k}, t) \\
& =t_{\alpha}^{<}(\mathbf{k}, t)-2 \Omega P_{\alpha \beta} \epsilon_{\beta z \gamma} u_{\gamma}(\mathbf{k}, t),
\end{aligned}
$$

where the $<$ symbol indicates that the nonlinear transfer terms are integrated over a truncated domain defined such that $\mathbf{p}+\mathbf{q}=\mathbf{k}$ with $256^{3}$ and $|\mathbf{q}|=q<k_{c}$. The eddy viscosity $\nu\left(k \mid k_{c}, t\right)$ is expressed as

$$
\nu\left(k \mid k_{c}, t\right)=-\iint_{\Delta^{>}} \theta_{k p q} \frac{S_{E_{2}}(k, p, q, t)}{2 k^{2} E^{V}(k, t)} d p d q .
$$

The function $S_{E_{2}}(k, p, q, t)$ corresponds to the so-called absorption term [linear in the energy spectrum $E(k, t)]$ in the EDQNM nonlinear transfer, lending itself in particular to an expression for the turbulent eddy viscosity, as is well known; $\Delta^{>}$is the integration domain over $(\mathbf{k}, \mathbf{p}$, and $\mathbf{q})$ triangles such that $p$ and/or $q$ are larger than $k_{c}$, and both $p$ and $q$ are smaller than $3 k_{c}$.

Finally, to take into account the effect of the emission (eddy noise) term involved in the EDQNM nonlinear transfer $\left[S_{E_{1}}(k, p, q, t)\right]$, we use a reconstruction field procedure, which enables us to partly rebuild the phase relationships between the three spectral components of the velocity field, as explained in detail in Paper II. $^{13}$ The functions $S_{E_{1}}(k, p, q, t)$ and $S_{E_{2}}(k, p, q, t)$ appearing in the transport coefficients used in the LES are written for completion in the Appendix. Note that although isotropic, the subgrid model we use in this paper has an important feature: it adjusts dynamically to the energy spectrum instead of assuming a given spectral law, usually the classical Kolmogorov law, $E(k) \sim k^{-5 / 3}$. This allows for the exploration of flows for which a theory to predict spectral indices is not available. Also note that the reconstruction procedure differs as well from traditional implementations insofar as it tries to keep some of the phase information of the small scales. 


\section{ROTATION AND ISOTROPY}

One of the effects of rotation on a flow is to induce anisotropy, as in the formation of large-scale columnar vortices. In that light, we explore in this section the anisotropic properties of a DNS at low Rossby number to see whether or not it is relevant to use a model based on isotropic assumptions to simulate a flow subjected to rotation. The LES model we propose to use approximates, as is customary, the transfer from the large to the small scales, but most of the modeled interactions are between small scales because of the value of $k_{c}$ (chosen to be in all cases larger than the energy injection wavenumber) and because most of the modes in a turbulent flow are in the small scales (recall that the number of modes in a given isotropic shell $k_{i}$ varies as $k_{i}^{2}$ ).

We therefore investigate now the properties of the small scales of flows forced with the TG vortex [see Eq. (6)] and subjected to rotation, with $k_{0}=2$ and at a Rossby number Ro $=0.03$; we perform a DNS on a grid of $256^{3}$ points and with the flow being initially at rest. To measure anisotropy, we introduce two different quantities, a spatial one and a spectral one, denoted respectively $I^{D}$ (for dimensional) and $I^{C}$ (for Craya; ${ }^{17}$ see also Ref. 18). Other measures of anisotropy ${ }^{19-21}$ are discussed later in Sec. IV B. The spatial coefficient $I^{D}$ evaluates the averaged ratio between the intensity of the velocity in the perpendicular direction $V_{\perp}(\mathbf{x}, t)$ and in the parallel direction $V_{\|}(\mathbf{x}, t)$, with $\perp, \|$ referring to the $z$-axis of rotation. The velocity field can be expressed as a function of these two components as $\mathbf{v}(\mathbf{x}, t)=V_{\|}(\mathbf{x}, t) \mathbf{e}_{\|}$ $+\mathbf{V}_{\perp}(\mathbf{x}, t)$, where $\mathbf{e}_{\|}$is the unit vector associated to the axis of rotation and $\mathbf{V}_{\perp}(\mathbf{x}, t)$ is the velocity field projected on the plane perpendicular to $\mathbf{e}_{\|}$.

The spatial anisotropy coefficient therefore reads

$$
I^{D}=\left\langle\frac{V_{\perp}(\mathbf{x}, t)}{V_{\|}(\mathbf{x}, t)}\right\rangle \text {. }
$$

The spectral coefficient $I^{C}$ is computed as in Ref. 22: for each wavevector $\mathbf{k}$, an orthonormal reference frame is defined as $\left[\mathbf{k} /|\mathbf{k}|, \mathbf{e}_{1}(\mathbf{k}) /\left|\mathbf{e}_{1}(\mathbf{k})\right|\right.$, and $\left.\mathbf{e}_{2}(\mathbf{k}) /\left|\mathbf{e}_{2}(\mathbf{k})\right|\right]$, with $\mathbf{e}_{1}(\mathbf{k})=\mathbf{k} \times \mathbf{z}$ and $\mathbf{e}_{2}(\mathbf{k})=\mathbf{k} \times \mathbf{e}_{1}(\mathbf{k})$, where $\mathbf{z}$ is the vertical unit wavevector. In that frame, since the incompressibility condition yields $\mathbf{k} \cdot \mathbf{v}(\mathbf{k})=0, \mathbf{v}(\mathbf{k})$ is only determined by its two components, $\mathbf{v}_{1}(\mathbf{k})$ and $\mathbf{v}_{2}(\mathbf{k})$. This second anisotropy coefficient is then defined as

$$
I^{C}=\sqrt{\left\langle\left|\mathbf{v}_{1}(\mathbf{k})\right|^{2}\right\rangle /\left\langle\left|\mathbf{v}_{2}(\mathbf{k})\right|^{2}\right\rangle} .
$$

Both $I^{D}$ and $I^{C}$ are such that they have unit values for fully isotropic flows.

In Fig. 1 we plot the temporal evolution of the total energy, the time being expressed in units of the eddy turnover time. Note that the long interval before turbulence fully develops, as rotation is strong and the run was started from a fluid at rest. Indeed, before the energy starts to grow at $t \approx 90$, one can observe a long transient during which the energy displays damped oscillations in time (see Paper I and Fig. 13 below). This transient is linked to the effect of rotation, and its duration increases linearly with $\Omega$, i.e., as the inverse of the Rossby number. During this first stage, the energy dissipation rate is small and the energy spectrum is

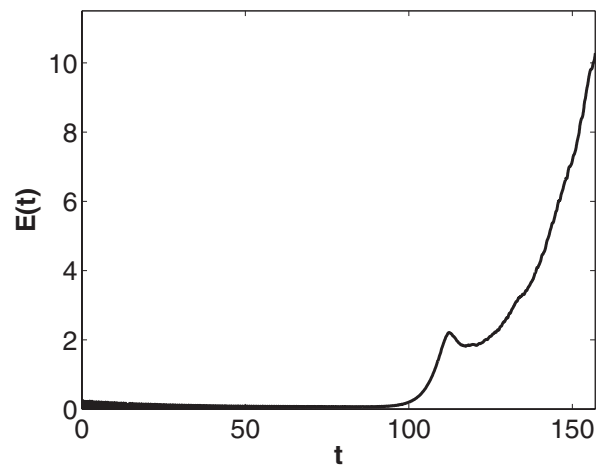

FIG. 1. Temporal evolution of the energy for the flow in a DNS with Ro $=0.03$; it displays two temporal phases, one dominated by wave interactions and one, for $t$ larger than 100, corresponding to an inverse cascade of energy.

very steep. Later, at $t \approx 90$, the enstrophy starts to grow and the energy dissipation rate increases. The energy also grows and an inverse cascade of energy develops. Turbulence sets in and the small-scale energy spectrum builds up an inertial range, with scaling close to $\sim k_{\perp}^{-2}$ (see Paper I for more details). In order to quantify the importance of anisotropy at what would be the subgrid scales in a LES of rotating flows, we start by noting that the velocity (in particular when an inverse cascade of energy develops at small enough Rossby number) is dominated by the large scales, whereas the modeling will occur in the small scales of the flow. In this context, we introduce a band-pass filter of the DNS data in order to concentrate the analysis on small-scale properties of the flow. The filtered field is given in Fourier space by all the velocity components at a wavevector $\mathbf{k}$ such that $32 \leq|\mathbf{k}|$ $\leq 64$; note that for this DNS using a classical 2/3 dealiasing rule, the maximum wavenumber is $k_{\max }=85$. As a result, the band-pass filter can be interpreted as preserving the small scales of the direct cascade inertial range.

Figure 2 represents the time history of the $I^{C}$ and $I^{D}$ anisotropy coefficients for the complete DNS (dashed line) and for the band-pass filtered velocity fields of the flow at Ro $=0.03$ (ovals). The Craya spectral coefficient $I^{C}$ of the complete DNS field remains close to unity during the whole simulation, indicating that globally the flow is close to an isotropic state. However, the directional coefficient $I^{D}$ exhib-
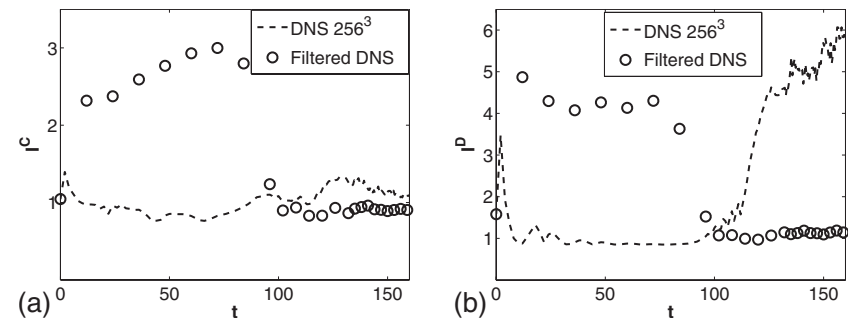

FIG. 2. Temporal evolution for a flow with Ro=0.03 of the Craya anisotropy coefficient $I^{C}$ (top) and the directional anisotropy coefficient $I^{D}$ (bottom) [see Eqs. (10) and (11)] for the full DNS velocity field (dashed line) and the filtered-DNS field (ovals) defined as a band-pass filter for wavevectors $|K| \in[32,64]$. Note the sharp transition toward isotropy of the small scales for $t \simeq 100$, as both the direct and inverse turbulence cascades finally develop. 
its three different regimes in the full DNS: a first phase between $t=0$ and $t \simeq 40$ during which it oscillates, with an amplitude that decreases with time, and a second phase between $t \simeq 40$ and $t \simeq 90$, with this coefficient remaining constant at a value close to unity, meaning that no direction is privileged in the flow. Finally, in a third and last phase, which begins when the turbulence starts to develop, $I^{D}$ strongly increases with time. This behavior is the signature of the generation of intense columnar structures within the flow, within which the perpendicular component of the velocity field dominates the parallel one.

The behavior of these coefficients is rather different for the filtered small-scale field, specially so for $I^{D}$. Indeed, the small scales are strongly anisotropic during the transient period before the turbulence develops, with a maximum value for $I^{C}$ of the order of 3 (and 5 for $I^{D}$ ). In this phase, the filtered directional anisotropy coefficient clearly shows that the perpendicular component of the velocity dominates the parallel one and therefore that the small scales are mostly bidimensional. At $t \simeq 80$, both coefficients drop rather abruptly to a value of order unity, indicating that when the turbulence develops, the small scales become isotropic, corresponding to a standard cascade of energy to small scales (note that the scales for which the anisotropic and inverse accumulation of energy takes place are eliminated by the band-pass filter).

With this study of the small-scale behavior of a flow subjected to moderate rotation, we see that an isotropic LES model cannot be used to treat every phase of the flow. Indeed, in the early transient phase, a model based on isotropic assumptions will not be able to approximate properly the transfer between the subgrid scales and the resolved scales (this point is discussed further in Sec. V). We therefore decide to only use our model to study the turbulent regime of rotating flows, after $t \approx 100$ in the case of Figs. 1 and 2 . Moreover, this is consistent with the fact that an isotropic LES is designed to study turbulent flows and cannot handle transitional (laminar and wave-dominated) flows. In the case of rotating flows starting from a fluid at rest, turbulence only develops after a transient time that depends linearly with the magnitude of the rotation. Note that in many studies, simulations of rotating flows are started from a previous turbulent steady state, and in that case our LES should have no problem to adapt as the spectral index changes with the evolution of the system.

Note that both coefficients $I^{C}$ and $I^{D}$ are relevant quantities in the context of this EDQNM-based LES: the behavior of $I^{C}$ justifies the assumption of "spectral isotropy" [i.e., dealing with $k$ instead of $\left(k_{\|}, k_{\perp}\right)$ at small scales]; on the other hand, the behavior of $I^{D}$ justifies the isotropic reconstruction done with the eddy noise because $I^{D} \approx 1$ is a measure of variance isotropy.

\section{NUMERICAL TESTS OF THE LES}

We now test our LES model against DNSs with different Rossby numbers. As stated before, the forcing used is the TG vortex [see Eq. (6)] at $k_{0}=2$. For each simulation, we follow the numerical procedure described in Paper I, namely, we
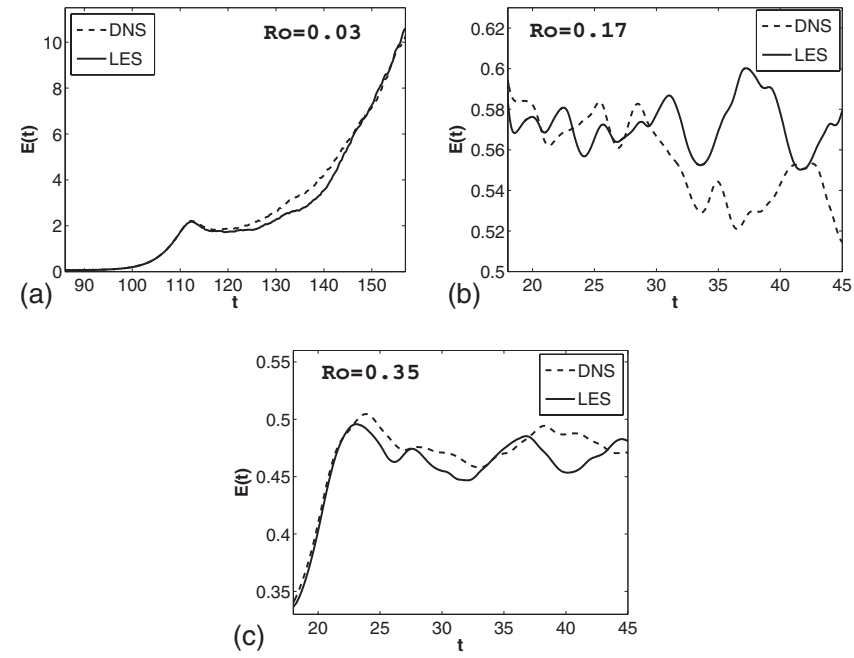

FIG. 3. Temporal evolution of the total energy for runs Id (DNS: $256^{3}$ ) and IL (LES: $64^{3}$ ) at Ro $=0.03$ (top), runs IId (DNS: $256^{3}$ ) and IIL (LES: $64^{3}$ ) at Ro $=0.17$ (middle), and runs IIId (DNS: 256 ${ }^{3}$ ) and IIIL (LES: $64^{3}$ ) at Ro $=0.35$ (bottom). DNS runs with dashed line; LES runs with solid line. Note the change in values on both axes for the low Rossby runs (top) because of the delay in the development of the turbulent phase when the LES is started and the ensuing accumulation of energy due to the inverse cascade now taking place at that low Rossby number.

vary the rotation rate leading to three different Rossby numbers: $0.03,0.17$, and 0.35 . The simulation parameters are summarized in Table I. The flow evolves in a periodic box, with $256^{3}$ grid points for the DNS and $64^{3}$ grid points for the LES. The "filtered-DNS" results in the table and figures refer to the filtered-DNS data on a grid of $64^{3}$ grid points, corresponding to the limited information contained in the LES grid. Since we are interested in studying only the modeling of the turbulent regime, we start the LES simulations from the filtered-DNS data at a time after the end of the transient phase. However, if the LES is started from a fluid at rest (i.e., started like the DNS at $t=0$ ), no significant differences are observed with the procedure of starting the LES at the end of the transient phase, except that the transient regime in the flow with Ro=0.03 is shorter. This accelerated evolution of the LES at low Rossby number during the transient when compared to the DNS can be easily explained considering the inclusion of transport coefficients in the LES, which assumes that a turbulent flow is already present.

\section{A. Global behavior of the flow}

The first test of the model is to examine the temporal evolution of the flow. This is displayed in Fig. 3 for the three Rossby numbers analyzed. The overall behaviors of the DNS and of the LES are similar in amplitude and in time scales. At intermediate Rossby numbers $(\mathrm{Ro}=0.17)$, the precise evolution of the DNS is not followed, although the energy obtained with the LES remains close to the DNS one. For the simulation at $\mathrm{Ro}=0.03$, an inverse cascade develops after $t \sim 120$, leading to a strong increase in the total energy. Although the LES model does not take wave interactions explicitly into account, it allows to reproduce this transfer of energy from the small scales to the very large ones with good accuracy; indeed, a simple scaling argument shows that in 

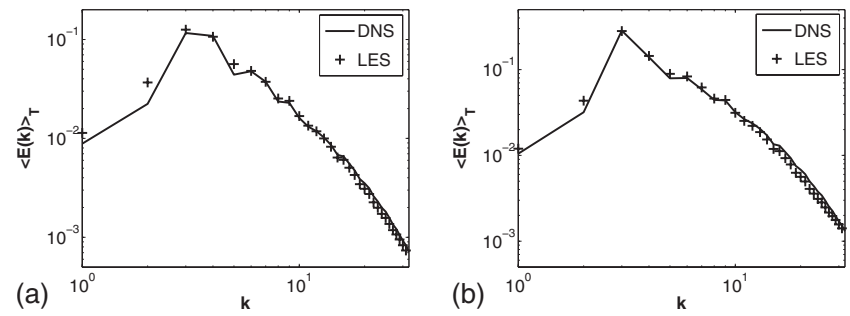

FIG. 4. Time averaged energy spectra for runs IId (DNS: $256^{3}$, solid line) and IIL (LES: $64^{3},++$ ) at Ro=0.17 (top) and runs IIId (DNS: $256^{3}$ ) and IIIL (LES: $64^{3}$ ) at $\mathrm{Ro}=0.35$ (bottom). Time averaging is performed from $t=25$ to $t=45$ for both simulations. Note the good agreement except possibly near $k=2$ corresponding to the forcing scale, indicative of a lack of adjustment of the LES at that scale, in particular for the perpendicular spectra (see Fig. 5).

the small scales, the eddy turn-over time is shorter than the time associated with waves and nonlinearities prevail. The LES is taking into account the interactions with the waves in an implicit way by changing the EDQNM time scale dynamically with the slope of the energy spectrum at large scales; this could be interpreted as "reversed" Kraichnan-like phenomenology. Note that the run at intermediate Rossby number has higher values of the energy because the forcing amplitude is larger than for the run at $\mathrm{Ro}=0.35$.

When looking at the time averaged isotropic energy spectra (see Fig. 4) for the two flows at the largest Rossby numbers, one can see that a good agreement is obtained. This figure also allows us to better understand the difference in the temporal evolution of the energy computed from the DNS and the LES data at Ro=0.17 (see Fig. 3). Indeed, although the model gives a good estimation of the DNS spectra at small scales, at very large scale (and particularly at $k=2$ ) non-negligible differences appear with the DNS, differences to which the total energy is sensitive. Note that a smaller difference between LES and DNS spectra can be observed at $k=2$ for the run at the higher Rossby number, $\mathrm{Ro}=0.35$. Otherwise, the spectrum is well approximated by the LES at all the other scales.

Similarly, when decomposing the energy spectra into their perpendicular and parallel components, a good agreement is reached at large scales, except again at $k=2$ for the perpendicular spectrum of the flow at Ro=0.17 (see Fig. 5). On the contrary, at small scales, the model seems to underestimate the spectra obtained by the DNS. This behavior is in fact due to the difference in resolution between the DNS and the LES: as $k_{\perp}$ and $k_{\|}$increase, the difference between the amount of modes taken into account in the evolution of these spectra for the DNS and for the LES increases as well. Note that the $k_{\|}$shells have the same number of modes independently of the value of $k_{\|}$(they are planes), while the number of modes in the $k_{\perp}$ shells grows as $k_{\perp}$ (modes are distributed in cylindrical shells), and this number grows as $k^{D-1}$ in dimension $D$ for isotropic (spherical) shells. We have checked that when making the comparison between the LES and the filtered DNS for instantaneous spectra, the discrepancy observed at high wavenumber disappears.

As mentioned earlier, the micro-Rossby-number measures how strong the imposed rotation is in the flow at the Taylor microscale, when compared to the rms vorticity de-
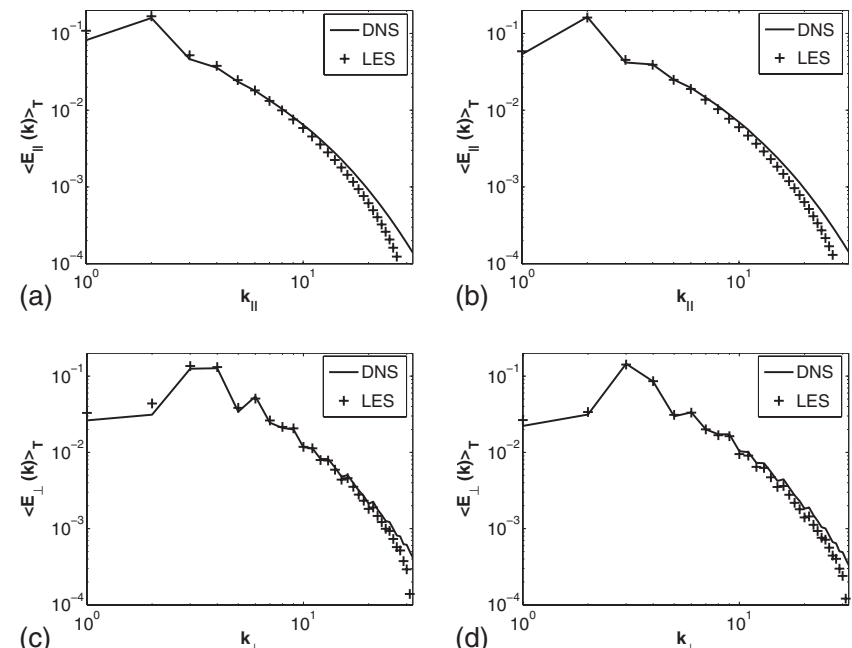

FIG. 5. Time averaged parallel (left) and perpendicular (right) energy spectra for runs IId (DNS: $256^{3}$, solid line) and IIL (LES: $64^{3},++$ ) at Ro $=0.17$ (top) and runs IIId (DNS: 256 ${ }^{3}$ ) and IIIL (LES: $64^{3}$ ) at Ro $=0.35$ (bottom).

veloped by the turbulence. Its time evolution is shown in Fig. 6 for all runs. Because the micro-Rossby-number emphasizes small scales that are not all present in an LES, $\mathrm{Ro}_{\omega}$ is also computed for the filtered DNS. We observe a good agreement between the truncated DNS and the LES, although the model slightly underestimates $\mathrm{Ro}_{\omega}$ for the two simulations at larger Rossby number. This behavior can be explained by enstrophy production in the LES, and whether it is associated to not strong enough eddy noise from subgrid scales to resolved scales associated or to too strong dissipation of energy due to the eddy viscosity (or both) is unclear for the moment. It may also be just a result of filtering and not preserving all small scales. Indeed, removing the subfilter scales in the LES leads to a smaller rms vorticity, which in turn can lead to the underestimation of the micro-Rossbynumber. Since LES are developed to model the evolution of the large eddies in the flow, it is not surprising that they are not as good at recovering microscale quantities.

In Table II we give the values of the characteristic parallel and perpendicular integral length scales (respectively, $L_{\|}$ and $L_{\perp}$ ) defined as

$$
\begin{aligned}
& L_{\|}=\frac{\int_{1}^{k_{\max }} E\left(k_{\|}\right) k_{\|}^{-1} d k_{\|}}{\int_{1}^{k_{\max }} E\left(k_{\|}\right) d k_{\|}}, \\
& L_{\perp}=\frac{\int_{1}^{k_{\max }} E\left(k_{\perp}\right) k_{\perp}^{-1} d k_{\perp}}{\int_{1}^{k_{\max }} E\left(k_{\perp}\right) d k_{\perp}},
\end{aligned}
$$

and computed at the final simulation time of each flow (note that the $k_{\|}=0$ mode is not included in the definition). Even if the values obtained by the LES data do not exactly correspond to the DNS values, they remain close; their differences can be explained by the same argument evoked before on the slight discrepancy between LES and DNS parallel and perpendicular energy spectra. Note that the perpendicular length scale is significantly larger for the lowest Rossby number, but the parallel length scales are comparable in all three runs. This is linked to the fact that the inverse cascade of energy, 

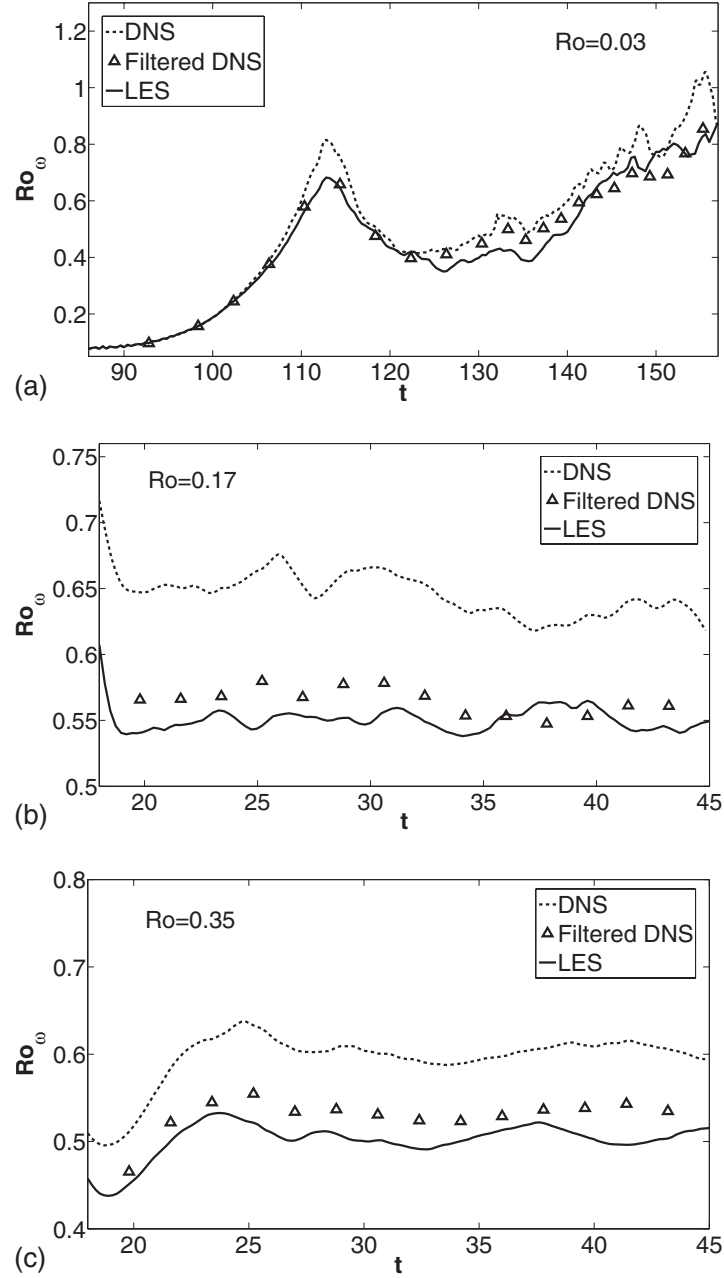

FIG. 6. Temporal evolution of the micro-Rossby-number $\mathrm{Ro}_{\omega}$ in flows with Ro=0.03 (top), Ro=0.17 (middle), and Ro=0.35 (bottom) for DNS on $256^{3}$ grid points (dashed line), filtered data of the DNS to a $64^{3}$ grid (triangles), and LES (solid line) on a $64^{3}$ grid. Note again the different scale on the axes, in particular for the lowest Rossby number in which case $\mathrm{Ro}_{\omega}$ approaches unity as the inverse cascade develops and energy and turbulence intensity grow.

which takes place at low Rossby number, is dominated by quasi-two-dimensional modes; the parallel spectrum does not undergo an inverse cascade, although energy does pile up at $k_{\|}=0$ mainly through resonant coupling of waves.

\section{B. Measures of anisotropy}

Rotating flows are known to develop anisotropies, and we now turn our attention to this point. In order to estimate the anisotropy of the different flows, we use the coefficients $I^{D}$ and $I^{C}$ defined earlier in Eqs. (10) and (11). They are shown as a function of time in Fig. 7 for the DNS (dashed line), the filtered-DNS data truncated to the LES resolution (triangles), and the LES (solid line) with $\mathrm{Ro}=0.03$. A very good match can be observed between the Craya coefficient $I^{C}$ computed from the filtered-DNS data and the one computed with the data from the LES model, whereas the coefficient computed with the full DNS data evolves on a lower level than the two other ones. This is due to the fact that the small scales of the field (i.e., scales with $k>k_{c}$ ) are taken into account in the spatial averaging process we perform to
TABLE II. Characteristic integral length scales $L_{\perp}$ and $L_{\|}$measured at different times $t_{m}$ for the three different Rossby numbers studied in this paper. Note that at the lowest Rossby number $(\mathrm{Ro}=0.03$, run $\mathrm{I})$, the perpendicular integral length scale is significantly larger than for more moderate rotation because of the inverse cascade.

\begin{tabular}{lcccc}
\hline \hline & & $t_{m}$ & $L_{\perp}$ & $L_{\|}$ \\
\hline Id & DNS & 157 & 5.73 & 2.99 \\
IL & LES & 157 & 5.63 & 2.95 \\
IId & DNS & 45 & 1.71 & 2.95 \\
IIL & LES & 45 & 1.74 & 3.22 \\
IIId & DNS & 45 & 1.76 & 2.69 \\
IIIL & LES & 45 & 1.83 & 2.71 \\
\hline \hline
\end{tabular}

calculate this coefficient. We saw in Sec. III that these small scales are more isotropic with a corresponding coefficient $I^{C}$ near unity, so when they are taken into account in the computation of the Craya coefficient, they lower its value. The small scales in the DNS are more isotropic, and as a result, the LES flow, which preserves a smaller amount of these scales, is globally more anisotropic and has a larger value of this coefficient.

As already observed in Fig. 2, the directional coefficient $I^{D}$ is strongly dominated by the large scales of the field, such as columnar structures appearing in the flow as a result of the inverse cascade process. Therefore, when we compare the
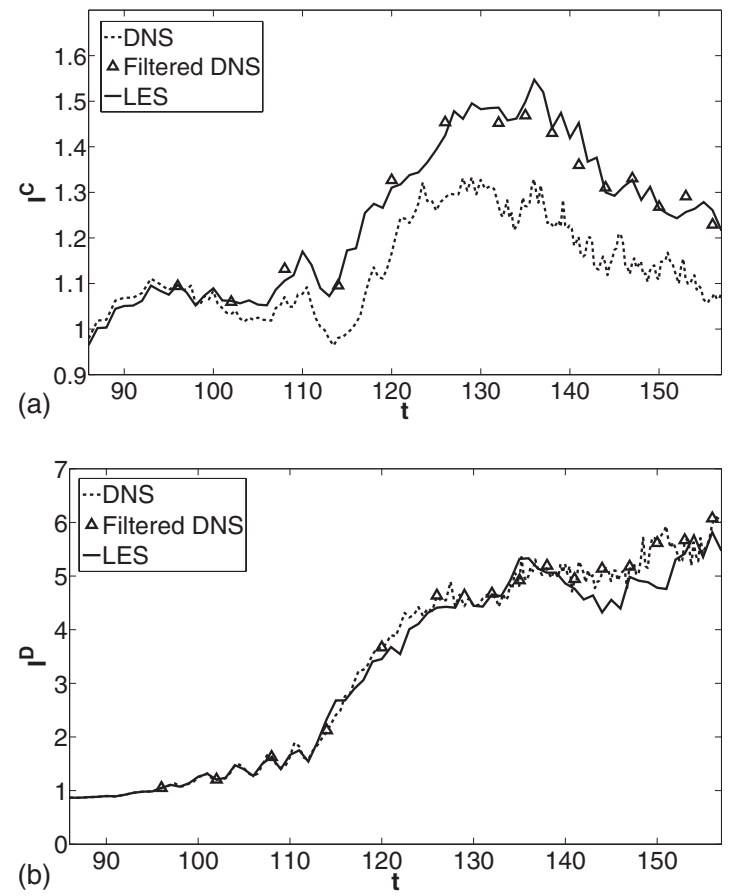

FIG. 7. Evolution of the spectral and directional isotropy coefficients $I^{C}$ and $I^{D}$ (see text) for runs I (full DNS, dashed line), Ir (filtered DNS: $64^{3}$, triangles), and IL (LES: $64^{3}$, solid line) at low Rossby number $(\mathrm{Ro}=0.03)$. Information about the cases at lower rotation rates is given in Table III. Isotropy is obtained when both coefficients are close to unity, and we note that the directional coefficient, related to real-space structures, indicates a stronger departure from isotropy than when measuring anisotropy in Fourier space as $I_{C}$ does [see Eqs. (10) and (11)] once the inverse cascade sets up and strong columnar vortices develop. Larger $I^{D}$ is also obtained for the runs at lower Rossby numbers (see Table III). 
TABLE III. Craya and directional isotropic coefficients $I^{C}$ and $I^{D}$ for the simulations at $\mathrm{Ro}=0.17$ and $\mathrm{Ro}=0.35$ at the final time of the computation.

\begin{tabular}{lccccc}
\hline \hline & & $t$ & Ro & $I^{C}$ & $I^{D}$ \\
\hline IId & DNS & 45 & 0.17 & 1.05 & 1.69 \\
IIr & Filtered DNS & $\ldots$ & $\ldots$ & 1.07 & 1.69 \\
IIL & LES & $\ldots$ & $\ldots$ & 1.07 & 1.71 \\
IIId & DNS & 45 & 0.35 & 1.04 & 1.97 \\
IIIr & Filtered DNS & $\ldots$ & $\ldots$ & 1.04 & 1.97 \\
IIIL & LES & $\ldots$ & $\ldots$ & 1.04 & 2.01 \\
\hline \hline
\end{tabular}

time history of this coefficient for the DNS and the filtered DNS, no noticeable difference appears. Once again our LES model predicts very well the evolution of this coefficient, even though the perpendicular component of the velocity clearly dominates over the parallel one. We also note that the model allows for a good estimation of both these coefficients for the simulations at larger Rossby numbers $(\mathrm{Ro}=0.17$ and Ro=0.35), as shown in Table III.

In our investigation of anisotropy of rotating flows, we now study the behavior of the $b_{i j}$ anisotropy tensor defined below; it is linked to the so-called "polarization" anisotropy introduced in Ref. 3 (see also Refs. 19, 23, and 24). This tensor, which is based on the Reynolds stress tensor $R_{i j}=\left\langle v_{i}(\mathbf{x}) v_{j}(\mathbf{x})\right\rangle$, is defined as

$$
b_{i j}=\frac{R_{i j}}{\Sigma_{l} R_{l l}}-\frac{\delta_{i j}}{3} .
$$

In Fig. 8 we represent the temporal evolution of the $b_{z z}$ component of the anisotropy tensor for runs I and III, at respectively $\mathrm{Ro}=0.03$ and $\mathrm{Ro}=0.35$. We first notice that the LES model predicts well the evolution of this coefficient for both simulations. Secondly, the development of a preferred direction in the flow at Ro= 0.03 (already observed in Fig. 7 through the increase in the directional coefficient $I^{D}$ in the inverse cascade) is also visible in this figure. Indeed, $b_{z z}$ tends to $-1 / 3$ as time increases since $v_{z}(\mathbf{x})$ becomes negligible when compared to the horizontal components $v_{x}(\mathbf{x})$ and $v_{y}(\mathbf{x})$.

Finally, we mention that other measures of anisotropy can be derived, as done for example in Refs. 20 and 21 where the authors examined specifically the behavior of the so-called dimensionality and circulicity tensors $D_{i j}$ and $F_{i j}$ defined as

$$
D_{i j}=\int \frac{k_{i} k_{j}}{k^{2}} v_{i} v_{j}^{*} d^{3} \mathbf{k}, \quad F_{i j}=\int \frac{\hat{\omega}_{i} \hat{\omega}_{j}^{*}}{k^{2}} d^{3} \mathbf{k},
$$

with ${ }^{*}$ denoting the complex conjugate as usual and $\hat{\omega}$ denoting the Fourier transform of the vorticity. Note that $\Sigma_{k} D_{k k}=\Sigma_{k} F_{k k}=\left\langle v^{2}\right\rangle$ and that defining $r, d$, and $f$ as the normalized forms of the $R, D$, and $F$ tensors $\left(t_{i j} \equiv T_{i j} / \Sigma_{k} T_{k k}\right)$ and with $b_{i j} \equiv r_{i j}$ in Eq. (14), we have $r_{i j}+d_{i j}+f_{i j}=\left\langle v^{2}\right\rangle \delta_{i j}{ }^{21}$ The dimensionality tensor is associated to the shape of eddies, whereas the circulicity tensor refers to the departure from mirror-symmetry of the flow since it relies on the vorticity.

We show in Fig. 9 the comparison between DNS and LES for the three diagonal components of the $d_{i j}$ (top) and of
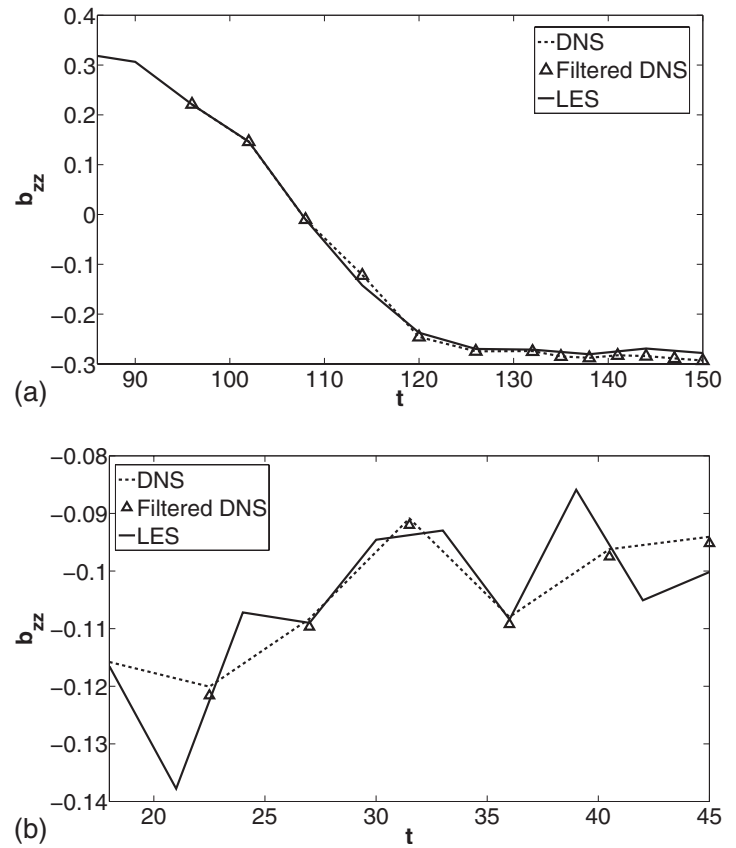

FIG. 8. Temporal evolution of the $b_{z z}$ component of the polarization anisotropy tensor [see Eq. (14)] for runs with Ro=0.03 (top) and Ro=0.35 (bottom), in the former case at late times once the pile-up of energy at large scales has begun. The DNS are plotted with a dashed line, the filtered DNS truncated to the resolution of the LES are given with triangles, and the solid line corresponds to the LES. Note again the tendency to evolve to a twodimensional state at late times, with $b_{z z} \rightarrow-1 / 3$, for the low Rossby number runs.

$f_{i j}$ (bottom) tensors, with directions 1-1, 2-2, and 3-3 given respectively with triangles, circles, and crosses and with the DNS in small symbols and the LES in large symbols. After the onset of the inverse cascade, strong departures from isotropy arise, and the model is much better at reproducing such departures when considering the coefficient based on the vorticity; we note however that when averaging over the horizontal plane, one recovers a good agreement between DNS and LES for both coefficients: on average, one captures the structures that develop with the model, but phases are not computed accurately (in particular, because of eddy noise), and thus the individual ( $x$ and $y$ ) components of the velocity vary widely between model and DNS (see also the discussion about the position of structures in physical space in Sec. IV E).

\section{Statistical analysis}

In this section, we investigate the statistics of the simulations at $\mathrm{Ro}=0.03$. Instantaneous probability density functions (PDFs) of the longitudinal and transverse derivatives of the $x$-component of the velocity field are computed and plotted in Fig. 10 at time $t=132$ in the inverse cascade. The PDFs computed on the full DNS data, the filtered DNS, and the LES agree well for the case of the longitudinal derivative. In the case of the transverse derivative, the DNS data differ from both the LES and the filtered-DNS data, the latter two displaying wider wings and being almost superimposed. It is well known that the small scales of a flow may have a strong influence on the distribution of velocity derivatives 

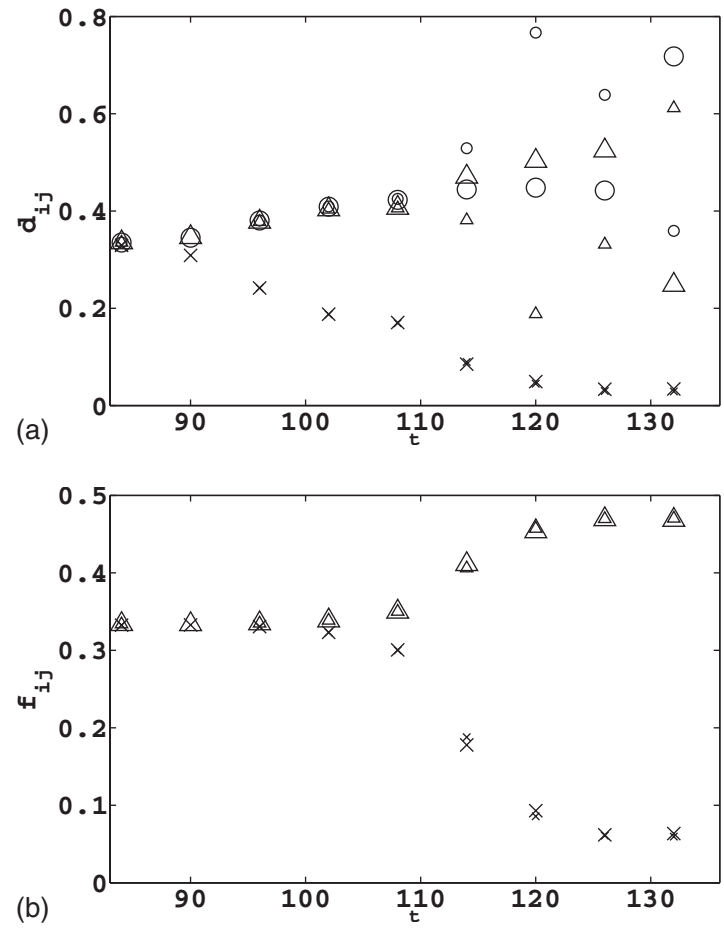

FIG. 9. Measuring anisotropy through the diagonal components of the tensors of dimensionality (top) and circulicity (bottom) [see Eq. (15)], with directions 1-1, 2-2, and 3-3 given respectively with triangles, circles, and crosses. Small symbols correspond to the DNS Ir and large symbols are for the LES Il at Ro=0.03. Note that the latter is much less scattered than the former for the components in the horizontal plane, and that it remains close to its isotropic value of $1 / 3$ for a longer time till the onset of the inverse cascade of energy, time after which anisotropy develops for both coefficients.

with strong velocity gradients appearing at small scale and that transverse derivatives show stronger tails in the PDFs (and therefore enhanced intermittency) than longitudinal derivatives. It is not clear whether this is the effect of more sensitivity to the intermittency in the transverse increments or whether it is the effect of anisotropy, but since the differences are stronger for the velocity derivatives taken in the direction of rotation, it may be attributed to anisotropies. Note that the PDFs of the full DNS data continue below the limit of $10^{-3}$ that we chose for the figure, and their wings become wider; however the PDFs of the LES and the filtered-DNS data do not extend below this limit since they sample a smaller range of values for the velocity gradients being at lower resolution.

In order to quantify the distributions of velocity fluctuations and the differences between DNS and LES data, we now compute the skewness $S_{k}$ of the longitudinal velocity derivative, i.e., its normalized third-order moment. The skewness, which measures the departure from Gaussian statistics, is usually negative for the longitudinal derivatives of a turbulent flow and oscillates around zero for the lateral ones. In Fig. 11 we show the time history of $S_{k}$. As for the energy, the LES model gives a correct prediction of the skewness for $86<t<145$, although around $t \simeq 140$, some discrepancies can be found that could be associated with the
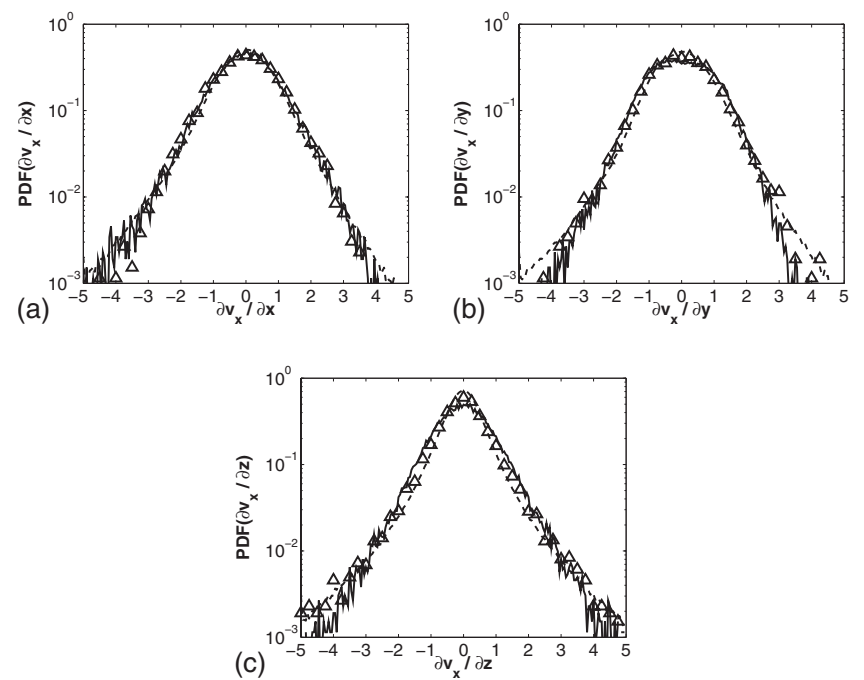

FIG. 10. PDFs of the velocity derivatives $\partial v_{x} / \partial x$ (top), $\partial v_{x} / \partial y$ (middle), and $\partial v_{x} / \partial z$ (bottom) for runs Id (DNS: $256^{3}$ ), Ir (filtered DNS: $64^{3}$ ), and IL (LES: $64^{3}$ ) at Ro=0.03 and $t=132$. As usual, the dashed line is for the full DNS flow, triangles for the filtered (truncated) DNS, and solid line for the LES.

development of structures. Note that this difference can be also associated with a slight discrepancy in total energy at around the same time (see Fig. 3).

\section{Intercomparison of models}

When evaluating LES approaches, it is useful to compare them to standard models one of which, for a spectral approach as used in this paper, is the CL model. ${ }^{14}$ We do so in this section, together with a comparison with an underresolved DNS at the same resolution as the LES and the CL simulations (same maximum wavenumber $k_{\max }$ ). We show in Fig. 12 (top) the results of this intercomparison with the normalized error for the energy spectrum defined as

$$
\mid E(k)_{\mathrm{DNS}}-E(k)_{\text {model }} / E(k)_{\mathrm{DNS}}
$$

averaged from $t=130$ to $t=150$ and the total enstrophy as a function of time (bottom). In both graphs, the model analyzed in this paper is drawn with a solid line, the DNS (or truncated DNS in the case of enstrophy) with a dashed line, the CL model with circles, and the under-resolved DNS with plus signs.

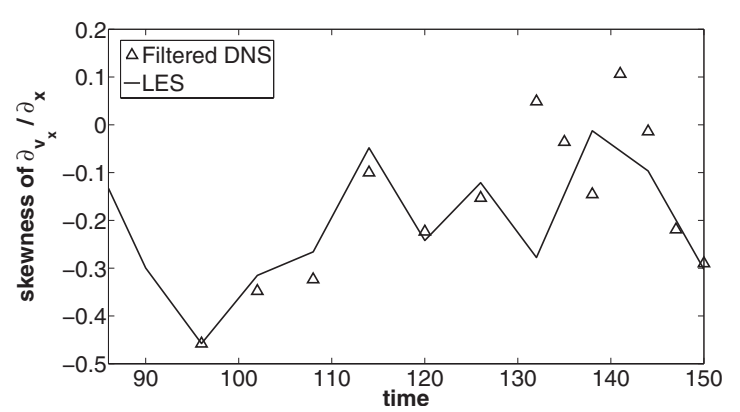

FIG. 11. Temporal evolution of the skewness for the longitudinal velocity derivative $\partial v_{x} / \partial x$ [see Eq. (7)] for runs Ir (filtered DNS: $64^{3}$, triangles) and IL (LES: $64^{3}$, solid line) at Ro=0.03. 

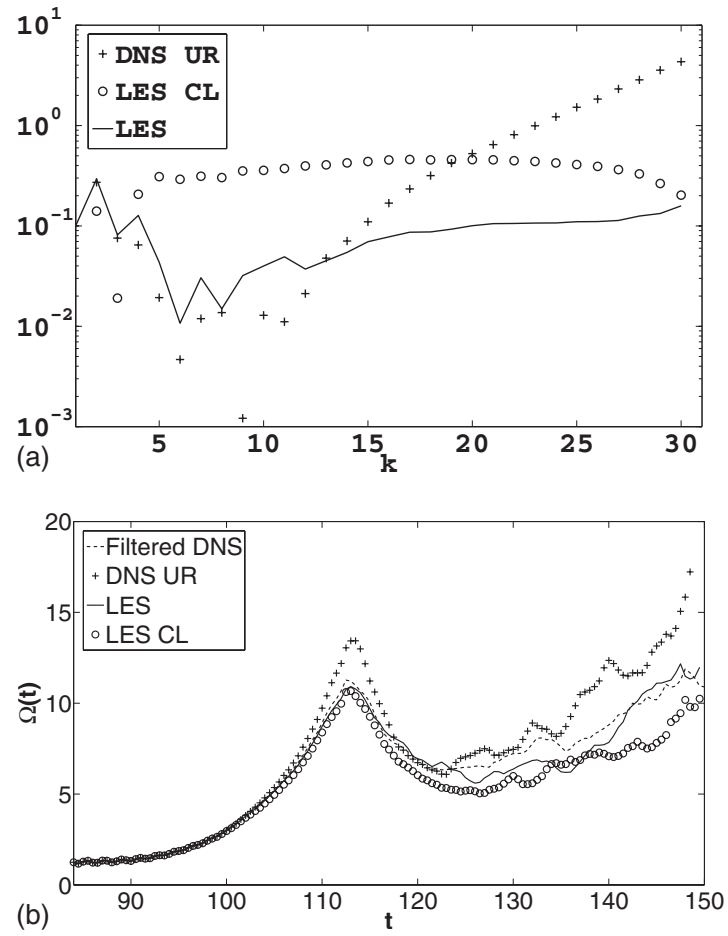

FIG. 12. Intercomparisons of a DNS with an under-resolved DNS (DNS UR, ++), our LES (solid line), and the CL model (LES CL, circles). Top: relative error in the energy spectra averaged from $t=130$ to $t=150$ [see Eq. (16)]. Bottom: temporal evolution of the total enstrophy, with the filtered DNS given with a dashed line.

Globally, the errors are smaller for the model presented in this paper. For example, the sum of errors for each method, for the energy spectra averaged over the time interval from $t=130$ to $t=150$, and as displayed in Fig. 12 (top) are respectively 11.8 for CL, 3.6 for our spectral model, and 22.7 for the under-resolved DNS; similar numbers are obtained for the total enstrophy. Note that at early times when inertial waves are prevalent (see Fig. 13), the models and the under-resolved DNS all give good results. The oscillatory phase, which can also be seen on the enstrophy (Fig. 12, bottom) ends shortly after $t \approx 100$ when the buildup of energy at large scale due to the inverse cascade begins and when the behavior between the different models begin as well. The good agreement between all runs in fact lasts until

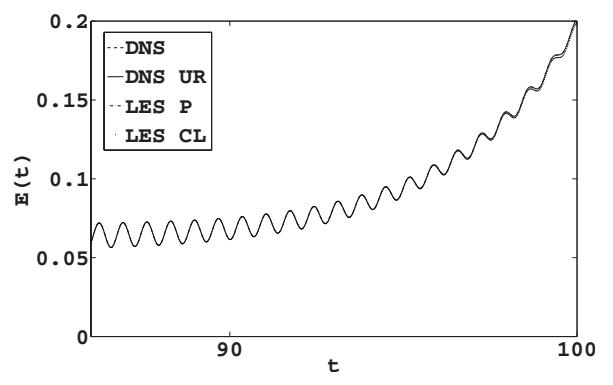

FIG. 13. Comparison between a DNS, an under-resolved DNS (DNS UR), our LES, and the CL model (LES CL) for a blow-up of the temporal evolution of the energy shortly after switching on the rotation; it shows the oscillatory behavior at early times and the good agreement of all models, which cannot be distinguished except at the very end when the CL model begins to depart slightly from the other runs.

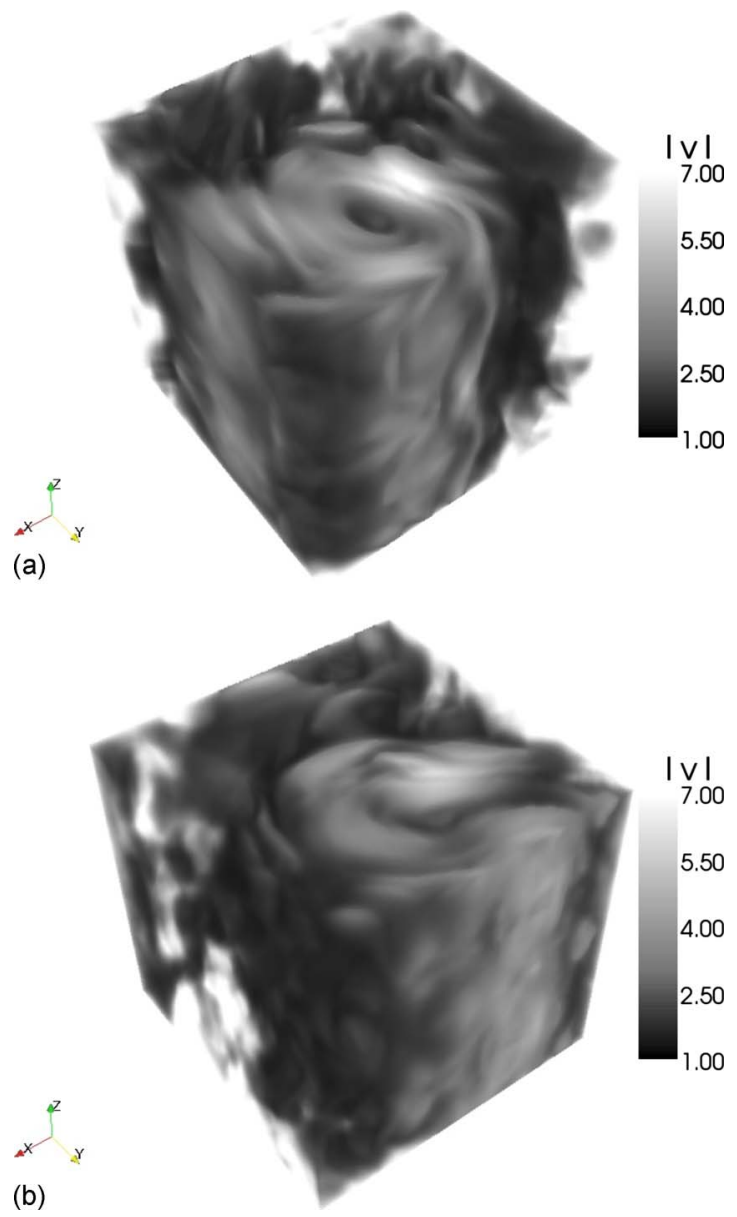

FIG. 14. (Color online) Volume rendering of the velocity modulus for flows at Ro $=0.03$-runs Ir (filtered DNS: $64^{3}$, top) and IL (LES: $64^{3}$, bottom) -at time $t=132$ for both simulations. The flow is dominated by a large eddy, but smaller vertical structures are visible as well.

$t \approx 112$ for the energy, the time when oscillations stop. We can conclude that overall, the under-resolved run is vastly outperformed by both LES, with a slight advantage to the model used in this paper over the CL model.

\section{E. Visualization in physical space}

We finally present a visualization in physical space of the velocity intensity at $t=132$ for the flow at $\mathrm{Ro}=0.03$. At this time of the simulation the inverse cascade already took place, and most of the flow energy was transferred to the $k_{\|}=0$ plane. We noted earlier that the TG flow injects no energy in the $k_{\perp}=1$ shell nor in the $k_{\|}=0$ shell. So all the energy we see at large scale is the result of an inverse cascade (in the former case) and of two dimensionalization (in the latter case). The evidence for the inverse cascade in this paper is given by the time evolution of the energy in Figs. 1 and 3 (see also Paper I, where fluxes are studied in detail). The accumulation of energy in this plane leads to the formation of columns as can be observed in Fig. 14. Although the structures are quasibidimensional, the isotropic LES model allows to reproduce them quite correctly. The spatial position differs slightly from the structure obtained by the DNS, but its size and intensity are well approximated. When examining the temporal evolution of the maximum of velocity (not 
shown), a good agreement occurs at all times. Note that this is a forced run visualized after $\approx 130$ turn-over times; as a result of the intrinsic sensitivity of turbulent flows due to their inherent unpredictability after a Lyapunov time of the order of a few turn-over times, the spatial position of the structures is not expected to be reproduced exactly by the LES. These differences in the position of the structures also explain the differences reported in Fig. 9.

\section{CONCLUSION}

We present in this paper a LES model for high Reynolds number rotating flows using a previously derived subgrid model $^{13}$ based on the isotropic EDQNM two-point closure with eddy viscosity and eddy noise. We show that, down to Rossby numbers of 0.03 , the small scales are sufficiently isotropic for the model to perform reasonably well. There are numerous laboratory experiments with which the comparison presented here could be extended, following the work in Ref. 8 using several EDQNM-based closures (see also Ref. 25 for the stratified anisotropic case). The advantage of using twopoint closures such as EDQNM as a model for turbulent flows in the presence of rotation is that it allows for building scaling laws at a relatively low computational cost and with the possibility of doing analytical estimations of nonlinear transfer (see, for example, Ref. 8). The model presented in this paper is much simpler since it is built on the isotropic three-dimensional version of the EDQNM; it is thus more limited in its scope insofar as it may not be able to explore very low Rossby numbers at moderate Reynolds numbers. On the other hand, following the standard LES methodology with spatially resolved large scales and turbulent coefficients to model the subgrid fluctuations, it allows to access more detailed features of the flows such as high-order statistics (e.g., PDFs) as well as spatial structures.

Also, the LES used in this paper adapts dynamically depending on the spectral index of the energy at superfilter (resolved) scales, and the values of the turbulent transport coefficients vary as a result. This is important in the context of rotating turbulent flows because the power law followed by the energy spectrum in this case is not quite ascertained yet and does vary with time. Phenomenological and theoretical predictions of this index, as well as several recent experiments, were reviewed in Ref. 15, with experimental and numerical evidence not quite able yet to sort out the different models or to fully describe the parameter space (e.g., as function of the rotation rate $\Omega$, the Reynolds number, etc.). An adequate LES model that can adjust to the resolved energy spectrum can help in this matter but more development and tests is needed. A reminiscent situation is found in magnetohydrodynamics when coupling the fluid to a magnetic field in the nonrelativistic limit; the total energy spectrum obtained analytically from the weak turbulence limit ${ }^{26,27}$ has been observed in the magnetosphere of Jupiter ${ }^{28}$ and in DNS, ${ }^{29}$ but the strong turbulence spectrum (or spectra in case there are different regions in parameter space) is a matter of debate.

However, the LES used in this paper does not adapt perfectly to all situations. We illustrate this point in Fig. 15,

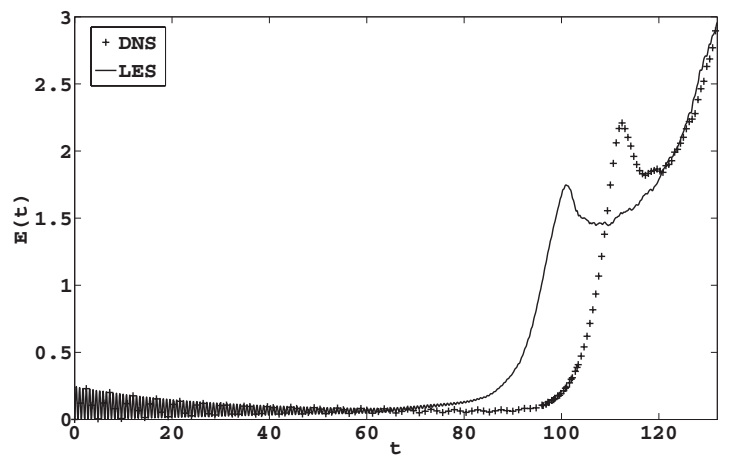

FIG. 15. Temporal evolution of the energy with the same LES run than in Fig. 3 (top), with a Rossby number Ro $\sim 0.03$ but now starting at $t=0$ as opposed to $t=84$ for Fig. 3 ; the DNS is displayed with ++ and the new LES run with a solid line. In this LES computation, the growth of energy occurs at an earlier time in the LES run, but the two runs become comparable at later times.

which presents the temporal evolution of the energy for the LES performed for the same data as the DNS analyzed, for example, in Fig. 3, but this time with the LES starting from $t=0$ as opposed to $t=84$. The oscillatory phases for both runs cannot be distinguished, but it is observed that the growth of energy due to the inverse cascade takes place sooner for the LES. Starting from zero initial conditions with the TG flow forcing, it can be checked that several low wavenumber modes grow substantially faster in this instantiation of the LES compared to the DNS. This may be related to the specific structure of the TG flow (see, e.g., Ref. 30) since no such acceleration of the inverse cascade of energy is observed when performing a similar experiment with other forcing functions, as, e.g., a Beltrami forcing. The TG flow has several mirror and rotational symmetries that result in very little excitation in some shells at early times. The turbulence nonlinear coupling is very slow to build up excitation in these modes in the DNS, whereas eddy noise and eddy viscosity contribute directly to their dynamics in the LES. The resulting LES flow can be viewed as somewhat artificially more turbulent, and therefore the inverse cascade starts earlier, as shown in Fig. 15. This problem can be alleviated by waiting for the turbulence coupling to develop in the flow; once all low wavenumber modes are filled up, the LES can be started with a turbulent flow.

Only one specific (nonhelical) forcing was explored in the DNS-LES comparisons studied in this paper. Further tests are required, considering other (nonhelical) forcing functions as well as forcing functions that introduce both energy and helicity in the flow. In the latter case, the implementation of the LES as described here may prove insufficient, and one should also consider taking into account the spectral properties and turbulent transport coefficients that include the effect of helicity, as done in the nonrotating case in Paper II. Such an implementation can also be of interest for nonhelical flows because even though helicity is not a positive definite quantity, local helical fluctuations develop rapidly in a flow through alignment of vorticity and pressure gradients. ${ }^{31}$ The properties of the model in the helical case in the presence of rotation will be dealt within a forthcoming paper. The freely decaying case (see Refs. 8, 11, 12, and 32 
for a global perspective) needs to be examined as well and is left for future work.

As a final remark, we want to stress the importance of developing adequate modeling of rotating (and stratified) flows, as encountered, for example, in the Earth's atmosphere. It was shown recently ${ }^{33}$ that the maximum intensity of a hurricane depends crucially on the (assumed) horizontal mixing length; this implies that an adequate treatment of the turbulence is essential in predicting various properties of hurricanes such as its intensity or landfall localization. A run with resolution down to $62 \mathrm{~m}$ shows strong local winds that were unresolved in previous studies. ${ }^{34}$ If the work presented here (as well as most of its predecessors) is far from reality for hurricane dynamical modeling (because of its lack of proper boundary conditions, stratification, and moisture), it nevertheless represents a first step toward the goal of a better understanding of geophysical flows, the issue here being that sufficiently high Reynolds number, i.e., sufficient multiscale interactions and two-way coupling between the small scales and the large scales in turbulent fluids supporting inertial (and/or gravity) waves, is a desired ingredient for testing LES approaches to geophysical turbulence.

\section{ACKNOWLEDGMENTS}

Computer time was provided by NCAR, which is sponsored by NSF, and by IDRIS (CNRS) under Grant No. 90597. P.D.M. is a member of the Carrera del Investigador Científico of CONICET.

\section{APPENDIX: CLOSURE EXPRESSIONS OF TRANSFER TERMS}

For completeness, we recall here the expression of the EDQNM closure equation for the kinetic energy spectrum $E(k, t)$ without helicity (note that the Coriolis term vanishes in the energy equation); it writes

$$
\left(\partial_{t}+2 \nu k^{2}\right) E(k, t)=\hat{T}(k, t)
$$

where the nonlinear transfer terms $\hat{T}(k, t)$ results from a convolution integral in Fourier space,

$$
\hat{T}(k, t)=\iint_{\Delta} \theta_{k p q}(t) S_{E}(k, p, q, t) d p d q .
$$

Here $\Delta$ is the integration domain with $p$ and $q$ such that $(\mathbf{k}, \mathbf{p}, \mathbf{q})$ form a triangle, and $\theta_{k p q}(t)$ is the relaxation time of the triple velocity correlations. As usual, ${ }^{35} \theta_{k p q}(t)$ is defined as

$$
\theta_{k p q}(t)=\frac{1-e^{-\left(\mu_{k}+\mu_{q}+\mu_{p}\right) t}}{\mu_{k}+\mu_{q}+\mu_{p}},
$$

where $\mu_{k}$ expresses the rate at which the triple correlations evolve, i.e., under viscous dissipation and nonlinear shear. It can be written as

$$
\mu_{k}=\nu k^{2}+\lambda_{K}\left(\int_{0}^{k} q^{2} E(q, t) d q\right)^{1 / 2} .
$$

Note that $\lambda_{K}$ is the only free parameter of the problem, taken equal to 0.36 to recover the Kolmogorov constant $C_{K}=1.4$ for a $k^{-5 / 3}$ classical energy spectrum. The expressions of $S_{E}(k, p, q, t)$ can be further described explicitly (with the time dependency of energy spectra omitted here) as

$$
\begin{aligned}
S_{E}(k, p, q, t) & =\frac{k}{p q} b\left[k^{2} E(q) E(p)-p^{2} E(q) E(k)\right] \\
& =S_{E_{1}}(k, p, q, t)+S_{E_{2}}(k, p, q, t) .
\end{aligned}
$$

Here, $S_{E_{1}}(k, p, q, t)$ and $S_{E_{2}}(k, p, q, t)$ are respectively used to denote the two terms of the extensive expression of $S_{E}(k, p, q, t)$. The geometric coefficient $b(k, p, q)$ (in short, $b$ in the previous expression) is defined as

$$
b(k, p, q)=\frac{p}{k}\left(x y+z^{3}\right),
$$

where $x, y$, and $z$ are the cosines of the inner angles opposite to $\mathbf{k}, \mathbf{p}, \mathbf{q}$.

${ }^{1}$ P. Sagaut and C. Cambon, Homogeneous Turbulence Dynamics (Cambridge University Press, Cambridge, 2008).

${ }^{2}$ S. Galtier, "Weak inertial-wave turbulence theory," Phys. Rev. E 68, 015301(R) (2003).

${ }^{3}$ C. Cambon and L. Jacquin, "Spectral approach to non-isotropic turbulence subjected to rotation," J. Fluid Mech. 202, 295 (1989).

${ }^{4}$ G. Holloway and M. Hendershott, "Stochastic closure for nonlinear Rossby waves," J. Fluid Mech. 82, 747 (1977).

${ }^{5}$ J. Bardina, J. H. Ferziger, and R. S. Rogallo, "Effect of rotation on isotropic turbulence: Computation and modeling," J. Fluid Mech. 154, 321 (1985).

${ }^{6} \mathrm{P}$. Bartello, O. Métais, and M. Lesieur, "Coherent structures in rotating three-dimensional turbulence," J. Fluid Mech. 273, 1 (1994).

${ }^{7}$ L. M. Smith, J. R. Chasnov, and F. Waleffe, "Crossover from two- to three-dimensional turbulence,” Phys. Rev. Lett. 77, 2467 (1996).

${ }^{8}$ C. Cambon, N. Mansour, and F. Godeferd, "Energy transfer in rotating turbulence," J. Fluid Mech. 337, 303 (1997).

${ }^{9}$ P. D. Mininni, A. Alexakis, and A. Pouquet, "Scale interactions and scaling laws in rotating flows at moderate Rossby numbers and large Reynolds numbers," Phys. Fluids 21, 015108 (2008).

${ }^{10}$ P. D. Mininni and A. Pouquet, "Helicity cascades in rotating turbulence," Phys. Rev. E 79, 026304 (2009).

${ }^{11}$ F. Bellet, F. S. Godeferd, J. F. Scott, and C. Cambon, "Wave turbulence in rapidly rotating flows," J. Fluid Mech. 562, 83 (2006).

${ }^{12}$ G. X. Cui, C. X. Xua, L. Fang, L. Shao, and Z. S. Zhang, "A new subgrid eddy viscosity model for large eddy simulation of anisotropic turbulence," J. Fluid Mech. 582, 377 (2007).

${ }^{13}$ J. Baerenzung, H. Politano, Y. Ponty, and A. Pouquet, "Spectral modeling of turbulent flows and the role of helicity," Phys. Rev. E 77, 046303 (2008).

${ }^{14}$ J. P. Chollet and M. Lesieur, "Parametrization of small scales of threedimensional isotropic turbulence utilizing spectral closures," J. Atmos. Sci. 38, 2747 (1981).

${ }^{15}$ C. Morize, F. Moisy, and M. Rabaud, "Decaying grid-generated turbulence in a rotating tank," Phys. Fluids 17, 095105 (2005).

${ }^{16}$ M. Bourgoin, R. Volk, N. Plihon, P. Augier, P. Odier, and J.-F. Pinton, "An experimental Bullard von Kármán dynamo,” New J. Phys. 8, 329 (2006).

${ }^{17}$ A. Craya, "Contribution à l'analyse de la turbulence associée à des vitesses moyennes," Ph.D. thesis, P.S.T. Ministère de l'air, 1958.

${ }^{18}$ J. Herring, Phys. Fluids 17, 859 (1974).

${ }^{19}$ Y. Morinishi, K. Nakabayashi, and S. Q. Ren, "Dynamics of anisotropy on decaying homogeneous turbulence subjected to system rotation," Phys. Fluids 13, 2912 (2001). 
${ }^{20}$ S. C. Kassinos, W. C. Reynolds, and M. M. Rogers, "One-point turbulence structure tensors," J. Fluid Mech. 428, 213 (2001).

${ }^{21}$ S. C. Kassinos, B. Knaepen, and D. Carati, "The transport of a passive scalar in magnetohydrodynamic turbulence subjected to mean shear and frame rotation," Phys. Fluids 19, 015105 (2007).

${ }^{22}$ J. H. Curry, J. R. Herring, J. Loncaric, and S. A. Orszag, "Order and disorder in two and three dimensional Bénard convection," J. Fluid Mech. 147, 1 (1984).

${ }^{23}$ S. Kida and J. Hunt, "Interaction between different scales of turbulence," J. Fluid Mech. 201, 411 (1989).

${ }^{24} \mathrm{X}$. Yang and J. A. Domaradzki, "Large eddy simulations of decaying rotating turbulence," Phys. Fluids 16, 4088 (2004)

${ }^{25} \mathrm{~F}$. Godeferd and C. Staquet, "Statistical modelling and direct numerical simulations of decaying stably stratified turbulence. Part 2. Large-scale and small-scale anisotropy," J. Fluid Mech. 486, 115 (2003).

${ }^{26}$ S. Galtier, S. V. Nazarenko, A. C. Newell, and A. Pouquet, "A weak turbulence theory for incompressible magnetohydrodynamics," J. Plasma Phys. 63, 447 (2000).

${ }^{27}$ S. Galtier, S. V. Nazarenko, A. C. Newell, and A. Pouquet, "Anisotropic turbulence of shear-Alfvén waves,” Astrophys. J. 564, L49 (2002).

${ }^{28}$ J. Saur, H. Politano, A. Pouquet, and W. H. Matthaeus, "Evidence for weak MHD turbulence in the middle magnetosphere of Jupiter," Astron. Astrophys. 386, 699 (2002).

${ }^{29}$ P. D. Mininni and A. Pouquet, "Energy spectra stemming from interactions of Alfvén waves and turbulent eddies," Phys. Rev. Lett. 99, 254502 (2007).

${ }^{30}$ M. E. Brachet, D. I. Meiron, S. A. Orszag, B. G. Nickel, R. H. Morf, and U. Frisch, "Small-scale structure of the Taylor-Green vortex," J. Fluid Mech. 130, 411 (1983).

${ }^{31}$ W. H. Matthaeus, A. Pouquet, P. D. Mininni, P. Dmitruk, and B. Breech, "Rapid directional alignment of velocity and magnetic field in magnetohydrodynamic turbulence," Phys. Rev. Lett. 100, 085003 (2008).

${ }^{32}$ J. Seiwert, C. Morize, and F. Moisy, "On the decrease of intermittency in decaying rotating turbulence," Phys. Fluids 20, 071702 (2008).

${ }^{33} \mathrm{G}$. Bryan and R. Rotunno, "The maximum intensity of tropical cyclones in axisymmetric numerical model simulations," Mon. Weather Rev. 137, 1770 (2009).

${ }^{34}$ Y. Chen, R. Rotunno, W. Wang, C. Davis, J. Dudhia, and G. J. Holland, "Large eddy simulations of an idealized hurricane," NCAR document (2008).

${ }^{35}$ M. Lesieur, Turbulence in Fluids (Kluwer-Academic, Dordrecht, 1997). 